\title{
3-Hydroxymethylpyridin, ein neues Stoffwechselprodukt der Mycobakterien *
}

\author{
D. Gross, A. Feige, A. Zureck und H.-R. Schütte \\ Institut für Biochemie der Pflanzen, Halle (Saale) und Institut für Mikrobiologie und experimen- \\ telle Therapie, Jena, der Deutschen Akademie der Wissenschaften \\ (Z. Naturforschg. 22 b, 835-838 [1967] ; eingegangen am 1. Februar 1967)

\begin{abstract}
Aus dem Kulturfiltrat von Mycobacterium bovis Stamm BCG wird neben Nicotinsäure eine weitere Bromcyan-positive Substanz isoliert und als 3-Hydroxymethylpyridin identifiziert. Dieses Pyridin-Derivat läßt sich auch als Ausscheidungsprodukt anderer Mycobakterien-Arten nachweisen. 3-Hydroxymethylpyridin ist eng mit dem Stoffwechsel der Nicotinsäure verbunden. Angebotene Nicotinsäure wird von den BCG-Bakterien aufgenommen und zu 3-Hydroxymethylpyridin umgewandelt.
\end{abstract}

Nach Untersuchungen von MotнEs wird von Mycobacterium bovis Stamm BCG neben Nicotinsäure eine weitere Bromcyan-positive Substanz gebildet und in das Nährmedium ausgeschieden ${ }^{1}$, deren Struktur und Bildung wir näher untersucht haben. Zur Isolierung der in ihrer Struktur bisher unbekannten Pyridinverbindung haben wir größere Mengen Kulturfiltrat eingeengt und bei $p_{\mathrm{H}} 9,5$ bis 10,0 ausgeäthert. Nach Abdestillieren des Äthers blieb eine gelbbraune Flüssigkeit zurück, die wir einer Gegenstromverteilung unterworfen haben. Als besseres Reinigungsverfahren erwies sich die Hochspannungs-Elektrophorese, weil sich die Nicotinsäure und die zu isolierende Pyridinverbindung durch unterschiedliches Wanderungsvermögen einwandfrei voneinander trennen ließen. Die Bromcyan-positiven Fraktionen wurden bei $p_{\mathrm{H}} 9,5-10,0$ ausgeäthert. Zur weiteren Reinigung haben wir den Ätherextrakt auf Kieselgel-H-Platten dünnschichtchromatographisch aufgetrennt. Die Elution erfolgte mit Äther. Die nach Abdestillieren des Äthers zurückbleibende Flüssigkeit wies im IR-Spektrum zwei starke Banden bei $1030 \mathrm{~cm}^{-1}(\mathrm{C}-\mathrm{OH})$ und bei $1600 \mathrm{~cm}^{-1}$ (Pyridinring) sowie eine breite Bande bei 3050 bis $3600 \mathrm{~cm}^{-1}(-\mathrm{OH})$ auf. An Hand der DMS-Kartei (Verlag Chemie, Weinheim/Bergstraße) konnte das Spektrum 3-Hydroxymethylpyridin zuge-

\footnotetext{
* Die physiologischen Teile dieser Arbeit sind der Dissertation A. Zureck, Mathematisch-Naturwissenschaftliche Fakultät der Ernst-Moritz-Arndt-Universität, Greifswald 1966, entnommen.

1 E. Mothes, Z. allg. Mikrobiol., Morphol., Physiol., Ökol. Mikroorganismen 4, 42 [1964].

* Herrn Dr. A. Kolbe, Institut für anorganische Chemie der Martin-Luther-Universität Halle-Wittenberg danken wir für die Aufnahme und Diskussion der IR-Spektren.

2 E. Dehnel, Chem. Ber. 33, 3498 [1900].
}

ordnet werden. Die IR- und UV-Spektren authentischer Testsubstanz und isolierter Pyridinverbindung waren identisch **.

Zur weiteren Charakterisierung wurden chromatographische Verfahren herangezogen. Das isolierte Produkt und 3-Hydroxymethylpyridin verhielten sich bei aufsteigender Papierchromatographie, zweidimensionaler Dünnschichtchromatographie und analytischer Gaschromatographie völlig gleich.

Pikrat, Schmp. $160-161^{\circ}$, und Hydrochlorid, Schmp. $116-119^{\circ}$, stimmten im Schmelzpunkt mit den in der Literatur angegebenen Werten ${ }^{2-7}$ überein. Die Mischschmelzpunkte ergaben keine Depression. Nach diesen chemisch-physikalischen Daten handelt es sich bei der aus Kulturfiltraten und Bakterienmaterial von $M$. bovis Stamm BCG isolierten Substanz um 3-Hydroxymethylpyridin, ein als Naturstoff bisher unbekanntes Pyridin-Derivat.<smiles>OCc1cccnc1</smiles>

(1)

Weitere Untersuchungen ergaben, daß 3-Hydroxymethylpyridin auch von anderen Arten der Gattung Mycobacterium wie $M$. tuberculosis, $M$. bovis, $M$. phlei, M. smegmatis, M. fortuitum, M. avium und

3 O. Jeger, J. Norymberski, S. Szpilfogel u. V. Prelog, Helv. chim. Acta 29, 684 [1946].

4 A. Cohen, J. W. Haworth u. E. G. Hughes, J. chem. Soc. [London] 1952, 4374.

5 K. W. Rosenmund u. F. Zymalkowski, Chem. Ber. 85, 152 [1952].

6 F. Bohlmann u. M. Bohlmann, Chem. Ber. 86, 1419 [1953].

7 H. S. Mosher u. J. E. Tessieri, J. Amer. chem. Soc. 73, 4925 [1951]. 
von unklassifizierten Arten gebildet wird. 3-Hydroxymethylpyridin tritt ebenso wie Nicotinsäure bei den einzelnen Arten in unterschiedlicher Menge auf. Diese Tatsache dürfte für eine biochemische Typendifferenzierung von großem Interesse sein. Entsprechende Arbeiten sind im Gange und werden an anderer Stelle veröffentlicht.

Das Auffinden von 3-Hydroxymethylpyridin als weitere Pyridinverbindung im Kulturfiltrat zeigt, daß die Farbreaktion beim Niacin-Test nach KonNo ${ }^{9,10}$ nicht nur durch Nicotinsäure hervorgerufen wird. Nach ersten Versuchen mit $M$. tuberculosis Stamm H $37 \mathrm{Rv}$ scheint die Gelbfärbung bei der $\mathrm{K}$ önig-Reaktion zum großen Teil durch 3-Hydroxymethylpyridin verursacht zu werden. Aus diesem Grund ist eine quantitative Nicotinsäurebestimmung mit der Bromcyan-Methode im Kulturfiltrat ohne chromatographische Auftrennung nicht möglich.

Nach Versuchen zur Biosynthese der Nicotinsäure entsteht diese bei $M$. bovis Stamm BCG ${ }^{1,11-14}$ und bei $M$. tuberculosis ${ }^{15-18}$ aus Asparaginsäure und einem $\mathrm{C}_{3}$-Körper (Glycerinanaloge) [vgl. Schema 1].

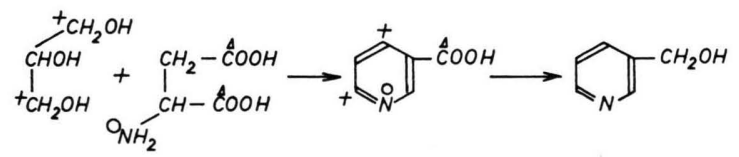

Schema 1. Biosynthese von Nicotinsäure und 3-Hydroxymethylpyridin.

In unseren früheren Versuchen ist den BCG-Bakterien ${ }^{14} \mathrm{C}$-markierte Asparaginsäure oder Glycerin$\left[{ }^{14} \mathrm{C}\right]$ angeboten und das Kulturfiltrat nach 10-tägigem Wachstum zur Isolierung der Nicotinsäure papierchromatographisch aufgetrennt worden. Die Radioaktivität fand sich auf den Papierchromatogrammen in der Nicotinsäurezone und in der Bande, die nach vorliegenden Untersuchungen 3-Hydroxymethylpyridin zuzuschreiben ist. Der Einbau von ${ }^{14} \mathrm{C}$-Aktivität in Nicotinsäure und 3-Hydroxymethylpyridin ließ eine biogenetische Verwandtschaft beider Pyridin-Derivate vermuten. Dieser Zusammen-

9 K. Konso, Science [Washington] 124, 985 [1956].

10 K. Konso, Beitr. Klin. Tuberkul. 119, 1 [1958].

11 E. Mothes, D. Gross, H. R. Schütte U. K. Mothes, Naturwissenschaften 48, 623 [1961].

12 D. Gross, H. R. Schütte, G. Hüвner u. K. Mothes, Tetrahedron Letters [London] 1963, 541.

13 D. Gross, A. Feige, R. Stecher, A. Zureck u. H. R. Schütte, Z. Naturforschg. 20 b, 1116 [1965]. hang ergab sich auch aus quantitativen Untersuchungen zur Bildung von Nicotinsäure und 3-Hydroxymethylpyridin bei BCG-Kulturen. BCG-Unterstämme, die viel Nicotinsäure bilden, produzieren in der Regel auch größere Mengen 3-Hydroxymethylpyridin. Ebenso geht auch die 3-Hydroxymethylpyridin-Bildung der Nicotinsäure-Produktion parallel (vgl. Tab. 1).

\begin{tabular}{|c|c|c|c|c|}
\hline \multirow[t]{2}{*}{$\begin{array}{l}\text { Kultur- } \\
\text { tage }\end{array}$} & \multicolumn{2}{|c|}{ Nicotinsäure } & \multicolumn{2}{|c|}{$\begin{array}{c}\text { 3-Hydroxymethyl- } \\
\text { pyridin }\end{array}$} \\
\hline & $\mu \mathrm{g} /$ Kultur* & $\underset{\text { Bakt.** }}{\mu \mathrm{g} / 20 \mathrm{mg}}$ & $\mu \mathrm{g} /$ Kultur* & $\begin{array}{l}\mu \mathrm{g} / 20 \mathrm{mg} \\
\text { Bakt.** }\end{array}$ \\
\hline 3 & 145,8 & 9,8 & 10,0 & 0,7 \\
\hline 5 & 399,5 & 19,9 & 23,7 & 1,2 \\
\hline 7 & 802,0 & 35,3 & 84,5 & 3,5 \\
\hline 10 & 1145,9 & 53,0 & 155,7 & 7,4 \\
\hline 12 & 1023,1 & 53,8 & 282,1 & 14,8 \\
\hline
\end{tabular}

Tab. 1. Nicotinsäure- und 3-Hydroxymethylpyridin-Bildung (Unterstamm Jena, S a u t o n - Nährlösung). * 20 ml Kulturfiltrat + Bakterienmasse. ${ }^{* *}$ Trockengewicht.

In weiteren Versuchen haben wir die Unterstämme Marburg und Paris 888 auf einer Nicotinsäure-haltigen S a u t o n - Nährlösung kultiviert und die Nicotinsäureaufnahme nach 10-tägiger Kultur bestimmt (vgl. Tab. 2).

\begin{tabular}{|c|c|c|}
\hline \multirow[b]{2}{*}{$\begin{array}{c}\text { BCG- } \\
\text { Unterstamm }\end{array}$} & \multicolumn{2}{|c|}{ Nicotinsäure } \\
\hline & $\begin{array}{l}\mu \mathrm{g} / 20 \mathrm{ml} \text { Nähr- } \\
\text { lösung zugesetzt }\end{array}$ & $\begin{array}{l}\mu \mathrm{g} / 20 \mathrm{ml} \text { Kultur- } \\
\text { filtrat + Bakt. Ma- } \\
\text { terial nach } 10 \text { Tagen }\end{array}$ \\
\hline Paris 888 & 0 & 228 \\
\hline & 800 & 555 \\
\hline & 2000 & 1148 \\
\hline Marburg & 0 & 1171 \\
\hline (Behring-Werke) & 800 & 1255 \\
\hline A.G.) & 2000 & 2012 \\
\hline
\end{tabular}

Tab. 2. Nicotinsäurebilanz in 10-tägiger Kultur ( $\mathrm{S}$ a u t o n Nährlösung) bei Nicotinsäurezusatz. Bakt. = Bakterien.

Es zeigte sich besonders bei dem schwachen Nicotinsäurebildner Paris eine der zugesetzten Nicotinsäuremenge proportionale Ausscheidung von 3-Hydroxymethylpyridin bei gleichzeitiger Abnahme des Nicotinsäuregehaltes. Diese Versuche deuten eine

14 D. Gross, Abh. dtsch. Akad. Wiss. Berlin, Kl. Chem., Geol., Biol., Jg. 1966, Nr. 3, S. 151, Akademie-Verlag, Berlin.

15 A. Nelles u. H. Herschman, Tuberkulosearzt 14, 813 [1960].

16 C. Rio Estrada u. H. Patino, J. Bacteriol. 84, 871 [1962].

17 A. G. Moat u. J. N. Albertson, Federat. Proc. 23, 528 [1964].

18 J. N. Albertson u. A. G. Moat, J. Bacteriol. 89, 540 [1965]. 
Umwandlung Nicotinsäure $\rightarrow$ 3-Hydroxymethylpyridin an.

Zur Überprüfung dieser Frage haben wir mit einer modifizierten S a u t o n - Nährlösung gearbeitet, in der Asparagin durch sekundäres Ammoniumphosphat ersetzt wurde. Unter diesen Kulturbedingungen werden weder Nicotinsäure ${ }^{1}$ noch $3-\mathrm{Hy}$ droxymethylpyridin ausgeschieden; es fehlt Asparagin als Precursor des Pyridinringes. Setzt man dieser Asparagin-freien Nährlösung aber Nicotinsäure zu, so ist bei Nicotinsäureaufnahme eine Ausscheidung von 3-Hydroxymethylpyridin festzustellen (vgl. Tab. 3). Dabei zeigt sich eine deutliche Abhängigkeit der 3-Hydroxymethylpyridin-Bildung von der vorgelegten Nicotinsäure-Konzentration.

\begin{tabular}{lccc}
\hline BCG- & \multicolumn{2}{c}{ Nicotinsäure } & $\begin{array}{c}3 \text {-Hydroxy- } \\
\text { methylpyridin }\end{array}$ \\
$\begin{array}{c}\text { Unter- } \\
\text { stamm }\end{array}$ & $\begin{array}{c}\mu \mathrm{g} / 20 \mathrm{ml} \mathrm{NL} \\
\text { zugesetzt }\end{array}$ & $\begin{array}{c}\mu \mathrm{g} / 20 \mathrm{ml} \mathrm{KF} \\
+ \text { Bakt. } \\
\text { nach 10 Tagen }\end{array}$ & $\begin{array}{c}\mu \mathrm{g} / 20 \mathrm{ml} \mathrm{KF} \\
+ \text { Bakt. } \\
\text { nach 10 Tagen }\end{array}$ \\
\hline Montreal & 0 & 0 & 0 \\
& 500 & 63,4 & 147,2 \\
& 1000 & 187,3 & 431,2 \\
Tokio & 2000 & 545,2 & 938,0 \\
& 0 & 0 & 0 \\
& 1000 & 208,0 & 125,6 \\
& 2000 & 452,8 & 378,0 \\
& & 734,4 & 883,1 \\
\hline
\end{tabular}

Tab. 3. Nicotinsäureumsatz und 3-HydroxymethylpyridinBildung in 10-tägiger Kultur (modifizierte $\mathrm{S}$ a u t o n - Nährlösung). $\mathrm{NL}=$ Nährlösung, $\mathrm{KF}=$ Kulturfiltrat, Bakt. = Bakterien.

Verfolgt man die Ausscheidung von 3-Hydroxymethylpyridin in Abhängigkeit von der Zeit, so zeigt sich, daß der Nicotinsäuregehalt der Kulturlösung ständig abnimmt und die Konzentration an 3-Hydroxymethylpyridin ansteigt. Diese Versuche lassen den Schluß zu, daß 3-Hydroxymethylpyridin von den BCG-Bakterien nur dann gebildet wird, wenn Nicotinsäure zur Verfügung steht. Diese Annahme haben wir in orientierenden Versuchen mit ${ }^{14} \mathrm{C}$-markierter Nicotinsäure überprüft. Den Unterstämmen Göteborg und Montreal wurden Nicotinsäure$\left[{ }^{14} \mathrm{COOH}\right]$ und Nicotinsäure- $\left[4.6-{ }^{14} \mathrm{C}\right]$ in normaler und in Asparagin-freier S a u t o n - Nährlösung angeboten. Nach 10-tägigem Wachstum konnte radioaktives 3-Hydroxymethylpyridin isoliert werden. Offenbar kann die Carboxylgruppe der Nicotinsäure

8 B. SAUton, C. R. hebd. Séances Acad. Sci. 155, 860 [1912]. 19 H. KNöLL, Z. Tuberkulose 101, 485 [1952]. von den BCG-Bakterien zur Hydroxymethylgruppe reduziert werden, wie in Schema 1 dargestellt ist. Eingehende Biosyntheseversuche zum Beweis einer spezifischen Inkorporation sind in Arbeit.

Die physiologische Bedeutung von Nicotinsäure und 3-Hydroxymethylpyridin im Stoffwechsel der Mycobakterien ist Gegenstand weiterer Untersuchungen.

Herrn Prof. Dr. H. KNöLL danken wir für großzügige Unterstützung und Herrn Prof. Dr. Dr. h.c. mult. K. Mотнеs für anregende Diskussionen.

\section{Material und Methodik}

\section{Anzucht der BCG-Stämme}

Als Versuchsobjekt diente Mycobacterium bovis Stamm BCG. Stammhaltung und Kultur erfolgten nach den im BCG-Institut, Jena, üblichen Methoden ${ }^{19,} 20$. Die für die Vor- und Hauptkultur verwendete SautonNährlösung ${ }^{8}$ besaß folgende Zusammensetzung:

$\begin{array}{lc} & {[\mathrm{g}]} \\ \text { L-Asparagin } & 4,0 \\ \text { Magnesiumsulfat } \cdot 7 \mathrm{H}_{2} \mathrm{O} & 0,5 \\ \text { Ferriammoniumcitrat } & 0,05 \\ \text { Dikaliumhydrogenphosphat } & 0,5 \\ \text { Citronensäure } & 2,0 \mathrm{mg} \\ \text { Glycerin } & 60 \mathrm{ml} \\ \text { Zinkchlorid } & 1,0 \mathrm{mg} \\ \text { Aqua dest. ad } & 1000 \mathrm{ml}\end{array}$

Der $p_{\mathrm{H}}$-Wert der Nährlösung betrug 7,0. Als Kulturgefäße benutzten wir 100-ml-E r l e n m e y e r-Kolben mit $20 \mathrm{ml} \mathrm{S}$ a ut o n-Nährlösung. Die modifizierte $\mathrm{S}$ a u t on - Nährlösung enthielt an Stelle von Asparagin 4,0 g sekundäres Ammoniumphosphat pro Liter.

Die verwendeten Kulturen der BCG-Unterstämme und der saprophytischen Mycobakterien-Arten stammten aus dem Institut für Mikrobiologie und experimentelle Therapie, Jena. Die pathogenen Stämme wurden im Hygiene-Institut der Friedrich-Schiller-Universität (Frau Dr. Gruhn), Jena, kultiviert.

\section{Nächweis und quantitative Bestimmung von Nicotin- säure und 3-Hydroxymethylpyridin}

Das nach dem Ernten der Bakterien anfallende Kulturfiltrat wurde im Rotationsverdampfer bei $70^{\circ} \mathrm{C}$ Badtemperatur und $15 \mathrm{~mm}$ Vakuum eingeengt und aufsteigend papierchromatographisch aufgetrennt (Schleicher \& Schüll-Papier $2043 \mathrm{~b}$, Laufmittel: wassergesättigtes n-Butanol). Zum Sichtbarmachen der Pyridinverbindungen haben wir $0,5 \mathrm{~cm}$ breite Längsstreifen der Papierchromatogramme 20 Min. einer Bromcyanatmosphäre ausgesetzt, anschließend mit einer 0,25-proz. alkoholi-

20 A. ZuREck, Dissertation, Ernst-Moritz-Arndt-Universität, Greifswald 1966. 
schen Benzidinlösung besprüht und nach dem Trocknen erneut mit Bromcyan behandelt. Nicotinsäure erschien als roter Fleck, $R_{f}=0,3$, 3-Hydroxymethylpyridin als rotvioletter Fleck, $R_{f}=0,73-0,76$. Nach Elution mit Wasser erfolgte Rechromatographie. Der Gehalt an 3-Hydroxymethylpyridin im wäßrigen Eluat wurde kolorimetrisch nach Farbreaktion mit Bromcyan bei $436 \mathrm{~nm}$ im Specol (Fa. Carl Zeiss, Jena) oder spektrophotometrisch bei $260 \mathrm{~nm}$ (Spektralphotometer VSU I, Fa. Carl Zeiss, Jena) ermittelt. Die den gemessenen Extinktionswerten zugehörigen Konzentrationen haben wir entsprechenden Eichkurven entnommen.

Zur Bestimmung der in den Bakterien verbliebenen Pyridinverbindungen (freie Nicotinsäure und 3-Hydroxymethylpyridin) wurde das von der Kulturlösung abgetrennte und zweimal mit dest. Wasser gewaschene Bakterienmaterial zerrieben und $16 \mathrm{Stdn}$. bei Raumtemperatur mit Aceton stehengelassen. Die weitere Aufarbeitung dieses Acetonextraktes erfolgte wie die des Kulturfiltrates.

\section{Isolierung von 3-Hydroxymethylpyridin}

Größere Mengen Kulturfiltrat wurden mit Natronlauge auf $p_{\mathrm{H}}$ 9,5-10,0 eingestellt und erschöpfend ausgeäthert. Nach Abdestillieren des Äthers wurde eine gelbbraune Flüssigkeit erhalten, von der wir einen Teil mit einer Gegenstromverteilungs-Apparatur (40 Einheiten) untersucht haben. Als Unterphase diente 0,1-n. Natriumcitrat-Salzsäure-Puffer $p_{H} 4$, als Oberphase Äther. Nach der Verteilung enthielten die Einheiten Nr. 1-7 angereichert 3-Hydroxymethylpyridin, das noch mit geringen Mengen Nicotinsäure verunreinigt war.

Eine einwandfreie Reinigung gelang durch Auftrennung an einer Hochspannungs-Elektrophorese (Elphor VaP, Fa. Bender \& Hobein, München) mit Benzer Trennkarton 230 bei einer Durchlaufzeit von 1,5-2 Stdn. (Spannung 0,7 kV, Stromstärke $220 \mathrm{~mA}$ ). Als Puffer diente 0,05- $n$. Natriumacetat, $p_{\mathrm{H}}$ 5,0. Das zur Kathode wandernde 3 -Hydroxymethylpyridin ließ sich

21 A. Murray, W. W. Foreman u. W. Langham, J. Amer. chem. Soc. 70, 1037 [1948]. in 9 von 40 aufgefangenen Fraktionen nachweisen und

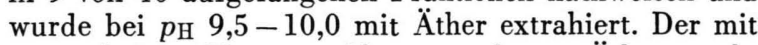
wasserfreiem Natriumsulfat getrocknete Ätherextrakt wurde eingeengt und auf Kieselgel H-Platten chromatographiert (Laufmittel: n-Propanol-Ammoniak, 10-proz., $95: 5)$. Die Elution erfolgte mit Äther.

\section{Identifizierung}

a) Das mit dem Zweistrahl-Spektralphotometer UR 10 (Fa. Carl Zeiss, Jena) aufgenommene IR-Spektrum zeigte starke Banden bei $1030 \mathrm{~cm}^{-1}$ und bei $1600 \mathrm{~cm}^{-1}$ sowie eine breite Bande bei $3050-3600$ $\mathrm{cm}^{-1}$. Im UV-Bereich (Spektralphotometer VSU I, Fa. Carl Zeiss, Jena) lag das Maximum bei $260 \mathrm{~nm}$.

b) Folgende chromatographische Verfahren dienten zur Identifizierung:

Aufsteigende Papierchromatographie (Schleicher \& Schüll-Papier 2043 b) mit wassergesättigtem n-Butanol als Laufmittel, $R_{f}=0,73-0,76$; zweidimensionale Dünnschichtchromatographie an Kieselgel G-Platten (Schichtdicke $25 \mu$ ). 1. Laufmittel: n-Propanol-Ammoniak (10-proz.) $95: 5, R_{f}=0,5$. 2. Laufmittel: Benzol-Methanol $4: 1, R_{f}=0,3$; Gaschromatographie (Panchromatograph, Fa. Pye \& Co., Cambridge) bei $135{ }^{\circ}$ C. Säule: $2,1 \mathrm{~m}, 4 \mathrm{~mm} \phi, 3 \%$ SE 30 auf silanisiertem Celit, 85-100 mesh.

c) Das aus methanolischer Lösung gefällte Pikrat zeigte nach mehrmaligem Umkristallisieren aus Wasser einen Schmelzpunkt von $160-161^{\circ} \mathrm{C}$. Aus ätherischer Lösung wurde durch Einleiten von $\mathrm{HCl}$ das Hydrochlorid gefällt und mit Äther gewaschen, Schmp. 116 bis $119^{\circ} \mathrm{C}$.

\section{5. ${ }^{14} \mathrm{C}$-markierte Verbindungen}

Die Darstellung von Nicotinsäure- $\left[{ }^{14} \mathrm{COOH}\right]{ }^{21}$ und Nicotinsäure- $\left[4.6-{ }^{14} \mathrm{C}\right]{ }^{22}$ erfolgte nach bekannten $\mathrm{Me}$ thoden. Die Messung der Radioaktivität haben wir unter dem Methandurchflußzähler (FH 49, Fa. Frieseke \& Hoepfner, Erlangen-Bruck) vorgenommen.

22 D. Gross, A. Feige u. H. R. Schütte, Z. Chem. 5, 21 [1965]. 